structions with proper cautions and restrictions will be observed.

A review of the foregoing study of our subject reminds us that while the field for lavage is large and its position as a therapeutic measure established, it requires skill and care to use it always for good and not for evil. There are many conditions, idiosyncrasies and circumstances which will forbid its use. Some of these can be overcome, some can not. Conservatism should mark the physician's course here, as in most departments of medical practice.

\section{THE EFFECT OF INHALATION OF OXYGEN} UPON THE HEMOGLOBIN.

Read in the Section on Practice of Medicine, at the Forty-sixth Annua Meeting of the American Medical Association, at Baltimore, Md., May 7-10, 1895.

BY GEORGE J. PRESTON, M.D.

PROFESSOR OF PIIYSIOLOGY AND DISEASES OF THE NERVOUS SYSTEM COLLEGE OF PHYSICIANS AND SURGEONS, BALTIMORE, MD.

Ever since the discovery of oxygen by Priestley in $\mathbf{1 7 7 4}$, it has been used as a therapeutic agent. In fact, Priestley himself made a number of experiments upon animals with the gas, thus preparing the way for experiments upon the human subject. A few years after his discovery, Lavoiser and Spalanzani demonstrated the relationship of oxygen to atmospheric air, and showed in a rough way the changes it produced in the blood. These experiments were more physiological than therapeutical, but not long after this date, Caillens in 1783 employed oxygen therapeutically. Jurine of Geneva, and Chaptal of Montpellier, followed with reports of cases of phthisis benefited by its use. The report of Fucroy to the French Academy was very extreme, making as it did absurd claims for oxygen in the treatment of numerous dis. eases. Beddoes in 1789 made a number of important observations upon the physiologic action of oxygen, and also employed it in practice. In conjunction with Sir Humphrey Davy and James Watt he established an institution for the treatment of disease by oxygen, an account of which may be seen in an extremely interesting paper on its therapentic use by Dr. A. H. Smith in the New York Medical Journal, April, 1870. Gardner in the American Journal of Medical Sciences, 1844, makes the remarkable statement that, " by the use of peroxid of nitrogen, or by rapid breathing, an increased quantity of oxygen enters, and as it can not be expelled as an element it acts upon more atoms of fibrin." Richardson made a number of experiments upon animals, and concludes that "the blood does not take up more oxygen unless more $\mathrm{CO}_{2}$ is given off." (Lancet, 1878.)

The above citations taken from the literature of the century following the discovery of oxygen, will give some idea of the way the profession regarded the subject. The literature is quite considerable but of little real value, since the rather crude experiments proved little, and the clinical experience related proved entirely too much. The experiments of Lav. oiser and Spalanzani referred to above, showed the effect of oxygen upon the blood, although the fact that blood exposed to the air changed color was known long before this time, as early as 1665 . Davy then extracted oxygen from blood; Regnault and Reiset and also Liebig announced that animals used the same amount of oxygen when breathing an atmos. phere composed of that gas alone as when they breathed ordinary air. (McKendric.) Liebig claimed that the gases were not merely dissolved in the blood, but existed in a state of loose chemic combination. Funke, Hoppe-Seyler, Schmidt, Stokes and others between the years 1853 and 1864 carefully worked out the nature and function of hemoglobin.

The history of the experiment of placing animals in an atmosphere of pure oxygen is an interesting one to physiologists, serving as a valuable object lesson. For many decades after the discovery of Priestley it was held that an animal could not live in this pure element. Then it was discovered that by providing for the absorption of the expired $\mathrm{CO}_{2}$ the life of the animal could be indefinitely prolonged. In regard to the number of respirations, my experiments showed that for the first few minutes there was a great rise, then a fail to about half the normal number. In the human subject the inhalation of pure oxygen seems to have little effect upon the pulse or arterial tension, but diminishes the number of respirations.

Admitting the fact that inhalations of pure oxygen under normal temperature and pressure, cause merely a diminution in the number of respirations a very practical question arises, namely, whether in the inspiration of pure oxygen the blood becomes more highly oxygenated than it does when ordinary air is breathed. The early observere fixed hardly any limit to the absorption of oxygen, believing it to be merely dissolved in the fluid as a whole. Then it was shown that the hemoglobin of the red corpuscle played a very important part, appropriating the oxygen from the plasma. As a matter of fact the serum is capable of absorbing little if any more of this element than of distilled water. The relationship which hemoglobin bears to the red corpuscle is still somewhat sub judice; we simply know that the combination of oxygen with hemoglobin is a very loose one, subject to certain laws of pressure and temperature, and that disassociation may be very easily brought about without disturbing the molecule of hemoglobin. (Foster.) The old observers thought that by the inhalation of pure oxygen a condition of what they termed "super saturation" of the blood was obtained. More exact observation showed this view to be untenable. Under pressure it is possible to make the serum take up more oxygen just as distilled water will do under like conditions. The quantity taken up by the hemoglobin of the red corpuscles is fixed, or nearly so. Experiments upon a solution of hemoglobin show that if a sufficient quantity of oxygen be present. each gram of the solution will take up $1.59 \mathrm{c}$. c. If there is an insufficient quantity present the hemoglobin will not be completely satisfied, or, to quote Foster: "We may perhaps say that a part only of the hemoglobin gets its allowance of oxygen, while the remainder continues reduced." Or it may very well be that while breathing ordinary air, a certain proportion of the red corpuscles pass through the pulmonary capillaries with their hemoglobin unsatisfied, or only partially satisnied with oxygen. The following experiments bear upon this point. The animals were placed under a glass jar which was first filled with pure oxygen which had been washed several times and a stream of the gas was allowed to pass in to the jar from a cylinder. A sufficient quantity of caustic potash was kept in the jar to absorb all the $\mathrm{CO}_{2}$. The hemoglobin was measured by the hemo. 
globinometer of Fleischl before and after the experiments.

Experiment 1. White rabbit; hemoglobin 58; respirations 100 . Was kept in the oxygen for five minutes, and at the end of this time the number of respirations had gone up to 200 a minute and there was no change in the per cent. of hemoglobin.

Experiment 2. Black rabbit; hemoglobin.52 ; respirations 100 . Was kept in oxygen for fifteen minutes. The number of respirations fell to 80 and the per cent. of hemoglobin rose to 80 .

Experiment 3. White rabbit; was kept in oxygen for thirty minutes and respirations dropped from 100 to 60 , and hemoglobin rose from 58 to 62 .

Experiment 4. Black rabbit; one hour in the oxygen; respirations dropped from 100 to 35 , and hemoglobin rose from 52 to 67 .

Experiment 5. White rabbit; thirty minutes in oxygen; respirations dropped from 120 to 56 and hemoglobin rose from 55 to 62 .

Experiment 6 . White rabbit; one hour in oxygen; respirations dropped only 10 , and there was no change in the hemoglobin. It was ascertained afterward that the oxygen in the cylinder was exhausted toward the close of the experiment.

Experiment 7 . White rat; respiration 200 ; hemoglobin 94. Kept in oxygen thirty minutes. Respiration dropped to 100 ; hemoglobin rose to 97.

of the seven experiments, there were only two that did not show diminution in the number of respirations and rise in the percentage of hemoglobin. Of the two failures Case 1 was not kept in the oxygen long enough, as later experiments proved, and the other, Case 6, through the exhaustion of oxygen in the cylinder was breathing practically atmospheric air toward the close of the experiment.

Experiment 8. Small dog. Oxygen was injected in to the rectum for forty minutes. Respirations fell from 24 to 10 ; hemoglobin rose from 82 to 84 .

In addition to the above experiments on animals, the following observations were made on four healthy men :

Experiment 9. Mr. C., weight 140 pounds ; inhaled oxygen for seven minutes; hemoglobin estimated before inhalation 82 ; after 92 .

Experiment 10. Mr. B., weight 160; time of inhalation five minutes; hemoglobin, before 78 ; after 87 .

Experiment 11. Mr. C., weight 150 ; time of inhalation six minutes; hemoglobin estimated before inhalation 76 ; after 83 .

Experiment 12. Dr. P., weight 190; inhaled oxygen five minutes; hemoglobin estimated before inhalation 80 ; after 95 .

There were no special symptoms attending the inhalation of oxygen except a certain lessening of the desire to breathe. There was not present the exhilaration and quickened pulse which has been described. This agrees with the observations made by Kerr, (Lancet, 1893). While one naturally hesitates to draw conclusions from so small a number of experiments, still it would seem reasonable that the inhalation of pure oxygen raises decidedly the percentage of hemoglobin in the blood. In all probability this is brought about, not by increasing the oxygen in the plasma, but by the fact that an excess of oxygen being present, all the hemoglobin is satisfied; or, to put it another way, fewer of the red corpuscles pass through the pulmonary capillaries, without obtaining their complement of oxygen.
While making these experiments it occurred to me that something might be learned from the study of the blood during and after the use of anesthetics. The first case examined showed a decrease of hemoglobin from 80 to 68 ; the estimations were made before the subject was put under chloroform and again after he had been under the influence of the anesthetic for fifteen minutes. Shortly after beginning this series of experiments I saw the very interesting paper by J.C. DaCosta, published in the Philadelphia Medical News, March 2, 1895. This observer demonstrated the marked reduction of hemoglobin during etherization, and also showed that this was not due to any diminution in the number of the red corpuscles. 'These experiments of DaCosta's taken together with my own, would go very far, it seems to me, toward proving the truth of the supposition stated in the first part of this paper, that in ordinary respiration a certain proportion of the red corpuscles were not completely satisfied with oxygen, or that in their passage through the pulmonary capillaries many of the corpuscles got through without having their reduced hemoglobin acted upon by oxygen.

The subject of the therapeutic use of oxygen, if any one will glance over the literature, has been treated in a thoroughly empirical manner. Almost every known disease has been cured by its use, if we can believe the glowing accounts of enthusiastic advocates. Moreover it has been so largely used by quacks, and so actively advertised by nostrum vendors, that the profession have naturally been deterred from its use. The investigation and the experiments related above were undertaken to see whether or not there was any scientific basis for the many claims made for the therapeutic use of oxygen. Although these experiments have been few, and not very exact, still enough has been done to show that the percentage of hemoglobin in the blood can be largely increased by the inhalation of pure oxygen. This method should have a full and scientific trial in pneumonia, acute bronchitis, pulmonary emphysema, pulmonary tuberculosis, and diseases obstructing the air passages. Again it might give good results in the treatment of certain forms of anemia. Its most brilliant future probably lies in its use by rectal injections. Kellogg and others have made some limited experiments with this method of introduction, but it needs further trial. Among those who have recorded clinical observations in the use of oxygen in various diseases are Gardner (American. Journal of Medical Sciences, 1844); Birch (Lancet, 1857 ) ; Richardson (Lancet, 1878); Professor Cabell (Virginia Medical Monthly, 1874); Oppenheim (Deutch Med. Woch., 1876); Hayem (Gaz. des Hop.); Gray (Virginia Medical Monthly, 1874); Wallian (N.Y. Med. Rec., 1883-84.85); (Med. News, 1890); Neech (British Medical Journal, 1892); Kerr (Lar.cet, 1893), and many others. For the full description of the methods of using oxygen therapeutically, reference is made to the interesting paper of Aulde in the Journal of the American Medical Association, Dec 6,1890 . These preliminary notes on the use of oxygen are offered in the hope that the profession may be induced to make further and more careful use of an agent which theoretically, at least, should prove valuable.

Blank applications for membership in the Association at the Journal office. 